\title{
Approximation by Szász Type Operators Involving Apostol-Genocchi Polynomials
}

\author{
Mine Menekşe Yılmaz \\ Department of Mathematics, Faculty of Arts and Science, University of Gaziantep, Gaziantep, TR-27310, Turkey \\ *Corresponding Author: Mine Menekşe Yllmaz. Email: menekse@gantep.edu.tr
}

Received: 07 May 2021 Accepted: 12 July 2021

\begin{abstract}
The goal of this paper is to give a form of the operator involving the generating function of Apostol-Genocchi polynomials of order $\alpha$. Applying the Korovkin theorem, we arrive at the convergence of the operator with the aid of moments and central moments. We determine the rate of convergence of the operator using several tools such as $\mathcal{K}$-functional, modulus of continuity, second modulus of continuity. We also give a type of Voronovskaya theorem for estimating error. Moreover, we investigate some results about convergence properties of the operator in a weighted space. Finally, we give numerical examples to support our theorems by using the Maple.
\end{abstract}

\section{KEYWORDS}

Apostol-Genocchi polynomials; rate of convergence; Korovkin theorem; modulus of continuity; Szász type operators; generating functions

\section{Introduction}

The Weierstrass approximation theorem shows that the polynomials are uniformly dense in the space of continuous functions on a compact interval equipped with supremum norm [1]. Polynomials are useful tools that are easy to evaluate, differentiate and integrate, and the Weierstrass theorem also shows their importance in the approximation theory. Since Bernstein [2] proved the Weierstrass theorem using a polynomial class in 1911, some authors [3-6], defined linear positive operators for the same purpose. One of these operators is Szász operators that generalization of Bernstein polynomials to infinite interval [7]:

$S_{n}(f ; x)=e^{-n x} \sum_{k=0}^{\infty} \frac{(n x)^{k}}{k !} f\left(\frac{k}{n}\right), \quad x \geq 0, n \in \mathbb{N}$,

Many mathematicians have found various generalizations of the Szász positive linear operator and studied the approximate behaviour of these new operators. The idea of establishing an operator using the generating function first appeared in [8]. In [8], assuming that $g(z)$ is analytic

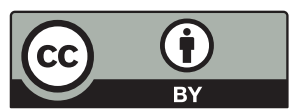

This work is licensed under a Creative Commons Attribution 4.0 International License, which permits unrestricted use, distribution, and reproduction in any medium, provided the original work is properly cited. 
function in the disk $|z|<r(r>1)$, the operator is defined as

$P_{n}(f ; x)=\frac{e^{-n x}}{g(1)} \sum_{k=0}^{\infty} p_{k}(n x) f\left(\frac{k}{n}\right)$,

where $p_{k}(x)$ are called Appell polynomails and their generating function is given by $g(t) e^{t x}=$ $\sum_{k=0}^{\infty} p_{k}(x) t^{k}$. The operators given by (2) are the most famous generalization of the Szász operators given in (1). After this paper, many authors have established new operators using both the polynomial and its generating function (see [9-19]). In all the mentioned studies, as a priority, the moments of the established operator were calculated, their approximate behaviour was examined, and also the speed of this approach was examined with the help of the modulus of continuity. What separates or connects these studies is the new operators set by a similar method. The Bohman-Korovkin theorems are a well-known method used to study the convergence problem of linear positive operators. Bohman-Korovkin theorems guarantee that a sequence of positive linear operators approaches uniformly to $f$ for each continuous function $f$. Checking the functions $1, x$ and $x^{2}$ are enough for this operation.

In [20], Apostol-Genocchi numbers and polynomials of (real or complex) order $\alpha, \alpha \in \mathbb{N} \cup\{0\}$, are defined with the help of the following generating functions, respectively

$$
\begin{aligned}
& \left(\frac{2 z}{\beta e^{z}+1}\right)^{\alpha}=\sum_{k=0}^{\infty} \mathcal{G}_{k}^{(\alpha)}(\beta) \frac{z^{k}}{k !}(|z|<|\log (-\beta)|), \\
& \left(\frac{2 z}{\beta e^{z}+1}\right)^{\alpha} e^{x z}=\sum_{k=0}^{\infty} \mathcal{G}_{k}^{(\alpha)}(x ; \beta) \frac{z^{k}}{k !}(|z|<|\log (-\beta)|),
\end{aligned}
$$

with $\mathcal{G}_{k}^{(\alpha)}(x):=\mathcal{G}_{k}^{(\alpha)}(x ; 1), \mathcal{G}_{k}^{(\alpha)}(\beta):=\mathcal{G}_{k}^{(\alpha)}(0 ; \beta), \mathcal{G}_{k}(x ; \beta):=\mathcal{G}_{k}^{(1)}(x ; \beta), \mathcal{G}_{k}(\beta):=\mathcal{G}_{k}^{(1)}(\beta)$, where $\mathcal{G}_{k}(\beta), \mathcal{G}_{k}^{(\alpha)}(\beta), \mathcal{G}_{k}(x ; \beta)$ denote the so-called Apostol-Genocchi number, Apostol-Genocchi number of order $\alpha$ and Apostol-Genocchi polynomial, respectively.

Generating functions for Apostol-Genocchi polynomials with their congruence properties involving these polynomials has been studied by many authors in recent years (see [21,22]).

Prakash et al. [23] established a sequence that includes Apostol-Genocchi polynomials of order $\alpha$, and then Deo et al. [24] introduced the Durrmeyer form of Apostol-Genocchi polynomials with Baskakov type operators. In this study, motivated by [23,24], we define a generalization of Szász type operators involving Apostol-Genocchi polynomials of order $\alpha$ as follows

$$
A_{n}^{(\alpha, \beta, m)}(f ; x)=e^{-(n+\eta) x}\left(\frac{2}{\beta e+1}\right)^{-\alpha} \sum_{k=0}^{\infty} \frac{\mathcal{G}_{k}^{(\alpha)}((n+\eta) x ; \beta)}{k !} f\left(\frac{k+m}{n+\eta}\right),
$$

where $f \in C[0, \infty)$ and $\mathcal{G}_{k}^{(\alpha)}(x ; \beta)$ is Apostol-Genocchi polynomials given in Eq. (4). The aim of this study is to give some convergence properties of Eq. (5). 


\section{Convergence of the Operator $A_{n}^{(\alpha, \beta, m)}$}

In this section, to begin with, we find the moments of the operator in Eq. (5) by using the generating function of Apostol-Genocchi polynomials. In addition, we prove the convergence of the operator $A_{n}^{(\alpha, \beta, m)}$ with the help of the moments.

Lemma 2.1. The operator $A_{n}^{(\alpha, \beta, m)}$ satisfies the following equalities:

$A_{n}^{(\alpha, \beta, m)}(1 ; x)=1$,

$A_{n}^{(\alpha, \beta, m)}(s ; x)=x+\frac{\alpha}{(n+\eta)(1+e \beta)}+\frac{m}{n+\eta}$,

$A_{n}^{(\alpha, \beta, m)}\left(s^{2} ; x\right)=x^{2}+\left(\frac{1+2 \alpha+e \beta}{(1+e \beta)}+2 m\right) \frac{x}{(n+\eta)}+\frac{1}{(n+\eta)^{2}}\left(\frac{\alpha^{2}-2 \alpha e \beta-\alpha e^{2} \beta^{2}}{(1+e \beta)^{2}}+\frac{2 m \alpha}{(1+e \beta)}+m^{2}\right)$.

Proof. By the aid of the generating function of the Apostol-Genocchi polynomials in Eq. (4), for Eqs. (6)-(8), we obtain

$\sum_{k=0}^{\infty} \frac{\mathcal{G}_{k}^{(\alpha)}((n+\eta) x ; \beta)}{k !}=\left(\frac{2}{e \beta+1}\right)^{\alpha} e^{(n+\eta) x}$,

$\sum_{k=0}^{\infty} \frac{\mathcal{G}_{k}^{(\alpha)}((n+\eta) x ; \beta)}{k !} k=\left(\frac{2}{e \beta+1}\right)^{\alpha} e^{(n+\eta) x}\left[(n+\eta) x+\frac{\alpha}{1+e \beta}\right]$,

$\sum_{k=0}^{\infty} \frac{\mathcal{G}_{k}^{(\alpha)}((n+\eta) x ; \beta)}{k !} k^{2}=\left(\frac{2}{e \beta+1}\right)^{\alpha} e^{(n+\eta) x}\left[(n+\eta)^{2} x^{2}+(n+\eta) x \frac{1+2 \alpha+e \beta}{1+e \beta}+\frac{\alpha^{2}-2 \alpha e \beta-\alpha e^{2} \beta^{2}}{(1+e \beta)^{2}}\right]$.

In view of Eqs. (9)-(11), we get the required result.

Remark 2.1. Using Lemma 2.1, we can give the central moments of the operator $A_{n}^{(\alpha, \beta, m)}$ as follows:

$A_{n}^{(\alpha, \beta, m)}(s-x ; x)=\frac{\alpha}{(n+\eta)(1+e \beta)}+\frac{m}{n+\eta}$.
$A_{n}^{(\alpha, \beta, m)}\left((s-x)^{2} ; x\right)=\frac{x}{n+\eta}+\frac{1}{(n+\eta)^{2}}\left[\frac{\alpha^{2}-2 \alpha e \beta-\alpha e^{2} \beta^{2}}{(1+e \beta)^{2}}+\frac{2 m \alpha}{1+e \beta}+m^{2}\right]$.

Theorem 2.1. If $f \in C[0, \infty)$, then $\lim _{n \rightarrow \infty} A_{n}^{(\alpha, \beta, m)}(f ; x)=f(x)$ uniformly on each compact subset of $[0, \infty)$.

Proof. We have fact that $\lim _{n \rightarrow \infty} A_{n}^{(\alpha, \beta, m)}\left(s^{i} ; x\right)=x^{i}, i=0,1,2$ from Lemma 2.1, and then we can use the Korovkin theorem to obtain the required result (see [25]). 


\section{The Rate of Convergence of the Operator $A_{n}^{(\alpha, \beta, m)}$}

The concept of modulus of continuity is the main instrument in approximation theory by positive linear operators. This concept works well in providing quantitive estimates. In this section, we use the usual modulus of continuity and the second modulus of continuity when measuring the rate of convergence. Since $\mathcal{K}$-functionals express some approximation properties of the function, we will also make use of the $\mathcal{K}$-functional when measuring the rate of convergence. We use the following notations throughout this paper. Let $C_{B}[0, \infty):=\{f \mid f:[0, \infty) \rightarrow \mathbb{R}$, $f$, is uniformly continuous and bounded function $\}$ with the norm $\|f\|_{C_{B}[0, \infty)}=\sup _{x \in[0, \infty)}|f(x)|$.

Definition 3.1. Let $f$ be uniformly continuous function on $[0, \infty)$ and $\delta>0$. The modulus of continuity $\omega(f ; \delta)$ of the function $f$ is defined by $\omega(f, \delta):=\sup _{\substack{x, y \in[0, \infty) \\|x-y| \leq \delta}}|f(x)-f(y)|$.

Then for any $\delta>0$, and each $x \in[0, \infty)$ the following relation holds

$|f(x)-f(y)| \leq \omega(f, \delta)\left(\frac{|x-y|}{\delta}+1\right)$.

Definition 3.2. The second modulus of continuity of $f \in C_{B}[0, \infty)$ is defined by

$\omega_{2}(f ; \delta):=\sup _{0<t \leq \delta}\|f(.+2 t)-2 f(t)+f(.)\|_{C_{B}[0, \infty)}$.

Definition 3.3. ([26]) The Peetre's $\mathcal{K}$-functional of the function $f \in C_{B}[0, \infty)$ is defined by

$\mathcal{K}(f ; \delta)=\inf _{h \in C_{B}^{2}[0, \infty)}\left\{\|f-h\|_{C_{B}[0, \infty)}+\delta\|h\|_{C_{B}^{2}[0, \infty)}\right\}$,

where $\delta>0$ and $C_{B}^{2}[0, \infty):=\left\{h \in C_{B}[0, \infty): h^{\prime}, h^{\prime \prime} \in C_{B}[0, \infty)\right\}$ with the norm

$\|h\|_{C_{B}^{2}[0, \infty)}:=\|h\|_{C_{B}[0, \infty)}+\left\|h^{\prime}\right\|_{C_{B}[0, \infty)}+\left\|h^{\prime \prime}\right\|_{C_{B}[0, \infty)}$.

It is well known that $\mathcal{K}$-functional and the second order modulus of continuity, $\omega_{2}(f ; \sqrt{\delta})$, are equivalent, i.e., there exists a constant $C>0$ such that for all $f \in C_{B}[0, \infty)$,

$\mathcal{K}(f, \delta) \leq C \omega_{2}(f ; \sqrt{\delta}), \quad \delta>0$,

(see [27]).

Theorem 3.1. If $f \in C_{B}[0, \infty) \cap W$, then $\left|A_{n}^{(\alpha, \beta, m)}(f ; x)-f(x)\right| \leq 2 \omega\left(f ; \delta_{n}\right)$, where $W=\left\{f: x \in[0, \infty)\right.$ and $\lim _{x \rightarrow \infty} \frac{f(x)}{1+x^{2}}$ exists and is finite $\}$ and $\delta_{n}(x)=\sqrt{A_{n}^{(\alpha, \beta, m)}\left((s-x)^{2} ; x\right)}$.

Proof. It follows from Lemma 2.1 and monotonicity property of operators $A_{n}^{(\alpha, \beta, m)}$ that $\left|A_{n}^{(\alpha, \beta, m)}(f ; x)-f(x)\right| \leq A_{n}^{(\alpha, \beta, m)}(|f(s)-f(x)| ; x)$. 
Using (14), we get the following from (19)

$\left|A_{n}^{(\alpha, \beta, m)}(f ; x)-f(x)\right| \leq \omega(f ; \delta)\left(1+\frac{1}{\delta} A_{n}^{(\alpha, \beta, m)}(|x-y| ; x)\right)$.

Applying the Cauchy-Schwarz inequality to the right side of (20), we get

$$
\left|A_{n}^{(\alpha, \beta, m)}(f ; x)-f(x)\right| \leq \omega(f ; \delta)\left(1+\frac{1}{\delta} \sqrt{A_{n}^{(\alpha, \beta, m)}\left((x-y)^{2} ; x\right)}\right) .
$$

By choosing $\delta:=\delta_{n}(x)=\sqrt{A_{n}^{(\alpha, \beta, m)}\left((x-y)^{2} ; x\right)}$ in (21), the proof is completed.

Definition 3.4. Let $f \in C_{B}[0, \infty)$ and $\alpha \in(0,1]$. The Lipschitz class of order $\alpha$ is defined as follows:

$\operatorname{Lip}_{M}(\alpha):=\left\{f \in C_{B}[0, \infty):|f(t)-f(x)| \leq M|t-x|^{\alpha} ; t, x \in[0, \infty)\right\}$,

where $M>0$.

Next theorem satisfies an estimate for the error of the operator $A_{n}^{(\alpha, \beta, m)}$ to a function $f$ belongs to Lipschitz class of order $\alpha$ by (22).

Theorem 3.2. Let $f \in \operatorname{Lip}_{M}(\alpha)$. For $x \in[0, \infty)$, we have the following inequality:

$\left|A_{n}^{(\alpha, \beta, m)}(f ; x)-f(x)\right| \leq M \delta_{n}^{\alpha}(x)$,

where $\delta_{n}(x):=\sqrt{A_{n}^{(\alpha, \beta, m)}\left((s-x)^{2} ; x\right)}$.

Proof. Since $A_{n}^{(\alpha, \beta, m)}$ is monotonic, we have

$\left|A_{n}^{(\alpha, \beta, m)}(f ; x)-f(x)\right| \leq M A_{n}^{(\alpha, \beta, m)}\left(|s-x|^{\alpha} ; x\right)$.

Using the Hölder inequality and from (24), we can write the following:

$\left|A_{n}^{(\alpha, \beta, m)}(f ; x)-f(x)\right| \leq M\left(A_{n}^{(\alpha, \beta, m)}\left((s-x)^{2} ; x\right)\right)^{\frac{\alpha}{2}}$.

Therefore, we obtain (23) by the help of the (25).

Theorem 3.3. Let $f \in C_{B}^{2}[0, \infty)$. The following inequality holds

$\left|A_{n}^{(\alpha, \beta, m)}(f ; x)-f(x)\right| \leq C \omega_{2}\left(f ; \sqrt{\tau_{n}}\right)+\omega\left(f ; \frac{\alpha}{(n+\eta)(1+e \beta)}+\frac{m}{n+\eta}\right)$,

where $C$ is a constant and $\tau_{n}=\frac{1}{4}\left[\left|A_{n}^{(\alpha, \beta, m)}\left((s-x)^{2} ; x\right)\right|+\left(\frac{\alpha}{(n+\eta)(1+e \beta)}+\frac{m}{n+\eta}\right)^{2}\right]$.

Proof. Assume that

$L_{n}(f ; x):=A_{n}^{(\alpha, \beta, m)}(f ; x)-f\left(x+\frac{\alpha}{(n+\eta)(1+e \beta)}+\frac{m}{n+\eta}\right)+f(x)$ 
and $g \in C_{B}^{2}[0, \infty)$. The expression

$g(s)=g(x)+(s-x) g^{\prime}(x)+\int_{x}^{s}(s-u) g^{\prime \prime}(u) d u$

is the Taylor expansion of $g$. If we apply the operator $L_{n}$ by (27) to both sides of the Eq. (28), and use the linearity property of the operator $L_{n}$, then we have the following:

$$
\begin{aligned}
\left|L_{n}(g ; x)-g(x)\right| & =\left|L_{n}\left(\int_{x}^{s}(s-u) g^{\prime \prime}(u) d u ; x\right)\right| \\
\leq & \left|A_{n}^{(\alpha, \beta, m)}\left(\int_{x}^{s}(s-u) g^{\prime \prime}(u) d u ; x\right)\right| \\
& +\left|\int_{x}^{x+\frac{\alpha}{(n+\eta)(1+e \beta)}+\frac{m}{n+\eta}}\left(x+\frac{\alpha}{(n+\eta)(1+e \beta)}+\frac{m}{n+\eta}-u\right) g^{\prime \prime}(u) d u\right| .
\end{aligned}
$$

Using (12) and (13) in (29), we get

$$
\begin{aligned}
& \leq \mid A_{n}^{(\alpha, \beta, m)}\left(\int_{x}^{s}(s-u) d u ; x\right)\left\|g^{\prime \prime}\right\|_{C_{B}[0, \infty)} \\
& +\left|\int_{x}^{x+\frac{\alpha}{(n+\eta)(1+e \beta)}+\frac{m}{n+\eta}}\left(x+\frac{\alpha}{(n+\eta)(1+e \beta)}+\frac{m}{n+\eta}-u\right) d u\right|\left\|g^{\prime \prime}\right\|_{C_{B}[0, \infty)} \\
& \leq\left[\left|A_{n}^{(\alpha, \beta, m)}\left((s-x)^{2} ; x\right)\right|+\left(\frac{\alpha}{(n+\eta)(1+e \beta)}+\frac{m}{n+\eta}\right)^{2}\right]\left\|g^{\prime \prime}\right\|_{C_{B}[0, \infty)}
\end{aligned}
$$

Using (17) in (30), we have

$\left|L_{n}(g ; x)-g(x)\right| \leq 4 \tau_{n}\|g\|_{C_{B}^{2}[0, \infty)}$,

where $\tau_{n}=\left|A_{n}^{(\alpha, \beta, m)}\left((s-x)^{2} ; \delta\right)\right|+\left(\frac{\alpha}{(n+\eta)(1+e \beta)}+\frac{m}{n+\eta}\right)$.

Now we consider the term of $A_{n}^{(\alpha, \beta, m)}(f ; x)-f(x)$.

$$
\begin{aligned}
\left|A_{n}^{(\alpha, \beta, m)}(f ; x)-f(x)\right|= & \left|L_{n}(f-g ; x)-(f-g)(x)\right| \\
& +\left|L_{n}(g ; x)-g(x)\right|+\left|f\left(\frac{\alpha}{(n+\eta)(1+e \beta)}+\frac{m}{n+\eta}\right)-f(x)\right| \\
\leq & 4\left[\|f-g\|_{C_{B}[0, \infty)}+\tau_{n}\|g\|_{C_{B}^{2}[0, \infty)}\right]+\omega\left(f ;\left|\frac{\alpha}{(n+\eta)(1+e \beta)}+\frac{m}{n+\eta}\right|\right) .
\end{aligned}
$$


When infimum is taken over all $g \in C_{B}^{2}[0, \infty)$ in (31), we obtain $\mathcal{K}$-functional given by (16) and the following inequality:

$\left|A_{n}^{(\alpha, \beta, m)}(f ; x)-f(x)\right| \leq 4 \mathcal{K}\left(f, \tau_{n}\right)+\omega\left(f ; \frac{\alpha}{(n+\eta)(1+e \beta)}+\frac{m}{n+\eta}\right)$.

Using Eq. (18) in (32), we obtain (26). Therefore the proof is completed.

Voronovskaya proved a theorem giving asymptotic error terms for the Bernstein polynomials for functions that can be differentiable twice (see [28]). Based on this idea, we present the following theorem.

Theorem 3.4. If $f \in C_{B}^{2}[0, \infty)$, then

$\lim _{n \rightarrow \infty}(n+\eta)\left[A_{n}^{(\alpha, \beta, m)}(f ; x)-f(x)\right]=\left(\frac{\alpha}{1+e \beta}+m\right) f^{\prime}(x)+\frac{f^{\prime \prime}(x)}{2 !} x$,

for every $x \geq 0$.

Proof. Let $f \in C_{B}^{2}[0, \infty)$ and $x \in[0, \infty)$ be fixed. From the Taylor formula for $f$ we have

$f(s)=f(x)+(s-x) f^{\prime}(x)+\frac{(s-x)^{2}}{2 !} f^{\prime \prime}(x)+r(s, x)(s-x)^{2}$,

where $r(s, x)$ is the Peano form of the remainder, $r(., x) \in C_{B}[0, \infty)$ and $\lim _{s \rightarrow x} r(s, x)=0$.

Applying the operator $A_{n}^{(\alpha, \beta, m)}$ to Taylor series of $f$ given by (34) and using the linearity of the operator $A_{n}^{(\alpha, \beta, m)}$, we have

$$
\begin{aligned}
(n+\eta)\left[A_{n}^{(\alpha, \beta, m)}(f(s) ; x)-f(x)\right]= & (n+\eta) f^{\prime}(x) A_{n}^{(\alpha, \beta, m)}(s-x ; x)+\frac{f^{\prime \prime}(x)}{2 !}(n+\eta) A_{n}^{(\alpha, \beta, m)} \\
& \times\left((s-x)^{2} ; x\right)+(n+\eta) A_{n}^{(\alpha, \beta, m)}\left(r(s, x)(s-x)^{2} ; x\right) .
\end{aligned}
$$

If we use the Cauchy-Schwarz inequality for the last term in (35), then we get

$A_{n}^{(\alpha, \beta, m)}\left(r(s, x)(s-x)^{2} ; x\right) \leq \sqrt{A_{n}^{(\alpha, \beta, m)}\left(r^{2}(s, x) ; x\right)} \times \sqrt{A_{n}^{(\alpha, \beta, m)}\left((s-x)^{4} ; x\right)}$.

Observe that $r^{2}(x, x)=0$ and $r^{2}(., x) \in C_{B}[0, \infty)$. Then it follows from Theorem 2.1 that

$\lim _{n \rightarrow \infty} A_{n}^{(\alpha, \beta, m)}\left(r^{2}(s, x) ; x\right)=r^{2}(x, x)=0$,

uniformly with respect to $x$ in every compact set of $[0, \infty)$. From (36) and (37), we have

$\lim _{n \rightarrow \infty}\left(A_{n}^{(\alpha, \beta, m)}\left(r^{2}(s, x) ; x\right)\right)^{1 / 2}(n+\eta)\left(A_{n}^{(\alpha, \beta, m)}\left((s-x)^{4} ; x\right)\right)^{1 / 2}=0$.

Moreover, if we use (12), (13) and (38) in (35), we get (33). This completes the proof. 


\section{Weighted Approximation for the Operator $A_{n}^{(\alpha, \beta, m)}$}

Let $\rho(x)=1+x^{2}$ be weight function. $B_{\rho}[0, \infty)=\left\{f:|f(x)| \leq M_{f} \rho(x), x \geq 0\right\}$, where $M_{f}$ is a constant depending on $f$. The weighted space $B_{\rho}[0, \infty)$ is normed linear space endowed with the norm $\|f\|_{\rho}=\sup _{x \in \mathbb{R}^{+}} \frac{|f(x)|}{\rho(x)}$. Let $C_{\rho}[0, \infty)$ be the set of continuous function in $B_{\rho}[0, \infty)$.

Lemma 4.1. If $f \in C_{\rho}[0, \infty)$ and $M>0$, then we have $\left\|A_{n}^{(\alpha, \beta, m)}(\rho ; x)\right\|_{\rho} \leq 1+M$.

Proof. In view of Lemma 2.1, we can obtain desired result.

Now, we have fact that the operators $A_{n}^{(\alpha, \beta, m)}$ maps from $C_{\rho}[0, \infty)$ to $B_{\rho}[0, \infty)$ thanks to Lemma 4.1.

Theorem 4.1. For every $f \in C_{\rho}[0, \infty) \cap W$, we have that $\lim _{n \rightarrow \infty}\left\|A_{n}^{(\alpha, \beta, m)}(f ; x)-f(x)\right\|_{\rho}=0$.

Proof. From [25], it is sufficient to verify the following cases:

$\lim _{n \rightarrow \infty}\left\|A_{n}^{(\alpha, \beta, m)}\left(s^{r} ; x\right)-x^{r}\right\|_{\rho}=0, \quad r=0,1,2$.

Since $A_{n}^{(\alpha, \beta, m)}(1 ; x)=1$, Eq. (39) is clear for $r=0$.

For $r=1$, using Lemma 2.1, we get

$\left\|A_{n}^{(\alpha, \beta, m)}(s ; x)-x\right\|_{\rho} \leq\left|\frac{\alpha}{(n+\eta)(1+e \beta)}\right| \sup _{x \in[0, \infty)} \frac{1}{1+x^{2}} \leq \frac{\alpha}{(n+\eta)(1+e \beta)}$

as $n \rightarrow \infty$ the condition in (39) holds. Similarly, we have

$\left\|A_{n}^{(\alpha, \beta, m)}\left(s^{2} ; x\right)-x^{2}\right\|_{\rho} \leq \frac{1}{(n+\eta)}\left(\frac{1+2 \alpha+e \beta}{1+e \beta}+2 m\right)+\frac{1}{(n+\eta)^{2}}\left(\frac{\alpha^{2}-2 \alpha e \beta-\alpha e^{2} \beta^{2}}{(1+e \beta)^{2}}+\frac{2 m \alpha}{1+e \beta}+m^{2}\right)$,

then, for $r=2$, the condition in Eq. (39) holds as $n \rightarrow \infty$. In view of (40) and (41), the proof is completed.

Corollary 4.1. For each $f \in C_{\rho}[0, \infty) \cap W$ and $a>0$, we have

$\lim _{n \rightarrow \infty} \sup _{x \in[0, \infty)} \frac{\left|A_{n}^{(\alpha, \beta, m)}(f ; x)-f(x)\right|}{\left(1+x^{2}\right)^{a+1}}=0$

Proof.

$\sup _{x \in[0, \infty)} \frac{\left|A_{n}^{(\alpha, \beta, m)}(f ; x)-f(x)\right|}{\left(1+x^{2}\right)^{1+a}} \leq \sup _{x \leq x_{0}} \frac{\left|A_{n}^{(\alpha, \beta, m)}(f ; x)-f(x)\right|}{\left(1+x^{2}\right)^{1+a}}+\sup _{x \geq x_{0}} \frac{\left|A_{n}^{(\alpha, \beta, m)}(f ; x)-f(x)\right|}{\left(1+x^{2}\right)^{1+a}}$
$\leq\left\|A_{n}^{(\alpha, \beta, m)}(f ; x)-f(x)\right\|_{C[0, \infty)}+\|f\|_{\rho} \sup _{x \geq x_{0}} \frac{\left.\mid A_{n}^{(\alpha, \beta, m)}\left(1+t^{2} ; x\right)-x\right) \mid}{\left(1+x^{2}\right)^{1+a}}+\sup _{x \geq x_{0}} \frac{|f(x)|}{\left(1+x^{2}\right)^{1+a}}$. 
Let's examine the terms in (42). From the Theorem 2.1, $\left\|A_{n}^{(\alpha, \beta, m)}(f ; x)-f(x)\right\|_{C[0, \infty)} \rightarrow 0$ as $n \rightarrow \infty$. For any fixed $x_{0}$, by Lemma 2.1, $\sup _{x \geq x_{0}} \frac{\left.\mid A_{n}^{(\alpha, \beta, m)}\left(1+t^{2} ; x\right)-x\right) \mid}{\left(1+x^{2}\right)^{1+a}} \rightarrow 0$ as $n \rightarrow \infty$. When we choose $x_{0}>0$ so large, $\sup _{x \geq x_{0}} \frac{|f(x)|}{\left(1+x^{2}\right)^{1+a}}$ can be made small enough. These facts complete the proof.

\section{Numerical Example for $A_{n}^{(\alpha, \beta, m)}$}

In this section we give some examples to obtain an upper bound for the error $f(x)-$ $A_{n}^{(\alpha, \beta, m)}(f ; x)$ in the terms of the modulus of continuity. The computations in this paper were performed by using Maple2021 ${ }^{\mathrm{TM}}$.

Example 5.1. Let $\beta=1, m=\eta=0$, and $n=0, \ldots, 7$. The approximation of $A_{n}^{(\alpha, \beta, m)}$ to $f=$ $\sin (\pi x)$ depends on $\alpha$ parameter on $[0, \infty)$ is shown in the Table 1 .

Table 1: The error estimation of $f(x)=\sin (\pi x)$ by using modulus of continuity

\begin{tabular}{llll}
\hline$n$ & Estimation for $\alpha=0$ & Estimation for $\alpha=1$ & Estimation for $\alpha=2$ \\
\hline 10 & 0.6180339888 & 0.5667047132 & 0.5237436440 \\
$10^{2}$ & 0.06282151816 & 0.06228435448 & 0.06183803480 \\
$10^{3}$ & 0.006283174972 & 0.006277800734 & 0.006273335408 \\
$10^{4}$ & 0.0006283185204 & 0.0006282647778 & 0.0006282201242 \\
$10^{5}$ & 0.00006283185306 & 0.00006283131564 & 0.00006283086910 \\
$10^{6}$ & 0.000006283185308 & 0.000006283179934 & 0.000006283175468 \\
$10^{7}$ & 0.0000006283185308 & 0.0000006283184770 & 0.0000006283184324 \\
\hline
\end{tabular}

Example 5.2. Let $\beta=1, m=\eta=1$ and $n=0, \ldots, 7$. The approximation of $A_{n}^{(\alpha, \beta, m)}$ to $f=$ $\sin (\pi x)$ depends on $\alpha$ parameter on $[0, \infty)$ is shown in the Table 2.

Table 2: The error estimation of $f(x)=\sin (\pi x)$ by using modulus of continuity

\begin{tabular}{ll}
\hline $\mathrm{n}$ & Estimation for $\alpha=1$ for $A_{n}^{(\alpha, \beta, m)}$ \\
\hline 10 & 0.5973816442 \\
$10^{2}$ & 0.06261992336 \\
$10^{3}$ & 0.006281178046 \\
$10^{4}$ & 0.0006282985716 \\
$10^{5}$ & 0.00006283165360 \\
$10^{6}$ & 0.000006283183314 \\
$10^{7}$ & 0.0000006283185108 \\
\hline
\end{tabular}

Example 5.3. The approximation of $A_{n}^{(\alpha, \beta, m)}$ to $f(x)=\frac{x^{3}}{\sqrt{1+x^{2}}}$ on $[0, \infty)$ for fixed $\beta=1, m=$ $\eta=0$, and $n=0, \ldots 7$, is shown in the Table 3 . 
Table 3: The error estimation of $f(x)=\frac{x^{3}}{\sqrt{1+x^{2}}}$ by using modulus of continuity

\begin{tabular}{llll}
\hline $\mathrm{n}$ & Estimation for $\alpha=0$ & Estimation for $\alpha=1$ & Estimation for $\alpha=2$ \\
\hline 10 & 0.3304906978 & 0.3040317266 & 0.2817895468 \\
$10^{2}$ & 0.0351254420 & 0.0348269498 & 0.0345789146 \\
$10^{3}$ & 0.0035332358 & 0.0035302160 & 0.0035277062 \\
$10^{4}$ & 0.0003535304 & 0.0003535002 & 0.0003534750 \\
$10^{5}$ & 0.0000353552 & 0.0000353548 & 0.0000353546 \\
$10^{6}$ & 0.0000035356 & 0.0000035356 & 0.0000035356 \\
$10^{7}$ & 0.0000003542 & 0.0000003542 & 0.0000003542 \\
\hline
\end{tabular}

\section{Conclusion}

In the present paper, we have introduced a form of the operator using the generating function of Apostol-Genocchi polynomials of order $\alpha$ and obtained the approximation properties and rate of convergence of this operator. At the end of the paper, we have found an upper bound for the error $f(x)-A_{n}^{(\alpha, \beta, m)}(f ; x)$ in the terms of the modulus of continuity for some functions. For further works, the approximation properties studied for Szász type operators involving ApostolGenocchi polynomials can also work for Kantorovich-Szász type operators involving ApostolGenocchi polynomials, moreover, the $q$ analogues of various modifications of Apostol-Genocchi polynomials of order $\alpha$ can discuss.

Acknowledgement: The author would like to thank the editor and referees for their many valuable comments and suggestions to improve the overall presentation of paper.

Funding Statement: The author received no specific funding for this study.

Conflicts of Interest: The author declares that he has no conflicts of interest to report regarding the present study.

\section{References}

1. Weierstrass, K. (1885). Über die analytische Darstellbarkeit sogenannter willkürlicher Functionen einer reellen Veränderlichen. Sitzungsberichte der Akademie zu Berlin, 2, 633-639.

2. Bernstein, S. (1912). Démonstration du théorème de Weierstrass fondée sur le calcul des probabilités. Communications of the Kharkov Mathematical Society, 13, 1-2.

3. Baskakov, V. A. (1957). An example of a sequence of linear positive operators in the space of continuous functions. Doklady Akademii Nauk SSSR, 113(2), 249-251.

4. Cheney, E. W., Sharma, A. (1964). Bernstein power series. Canadian Journal of Mathematics, 16, 241-252. DOI 10.4153/CJM-1964-023-1.

5. Meyer-König, W., Zeller, K. (1960). Bernsteinsche potenzreihen. Studia Mathematica, 19(1), 89-94. DOI 10.4064/sm-19-1-89-94.

6. Pethe, S. (1983). On linear positive operators. Journal of the London Mathematical Society, 2(27), 55-62. DOI 10.1112/jlms/s2-27.1.55.

7. Szász, O. (1950). Generalization of S. Bernstein's polynomials to the infinite interval. Journal of Research of the National Bureau of Standards, 45(3), 239-245. DOI 10.6028/jres.045.024. 
8. Jakimovski, A., Leviatan, D. (1969). Generalized Szász operators for the approximation in the infinite interval. Mathematica (Cluj), 11(34), 97-103.

9. Ismail, M. E. H. (1974). On a generalization of Szász operators. Mathematica (Cluj), 16(39).

10. Ispir, N., Atakut, Ç. (2002). Approximation by modified Szász-Mirakjan operators on weighted spaces. Proceedings of the Indian Academy of Sciences: Mathematical Sciences, 112(4), 571-578. DOI 10.1007/BF02829690.

11. Kajla, A., Agrawal, P. (2015). Approximation properties of Szász type operators based on Charlier polynomials. Turkish Journal of Mathematics, 39(6), 990-1003. DOI 10.3906/mat-1502-80.

12. Mursaleen, M., Ansari, K. J. (2015). On Chlodowsky variant of Szász operators by Brenke type polynomials. Applied Mathematics and Computation, 271(5), 991-1003. DOI 10.1016/j.amc.2015.08.123.

13. Sucu, S., Büyükyazc, I. (2012). Integral operators containing Sheffer polynomials. Bulletin of Mathematical Analysis and Applications, 4(4), 56-66.

14. Içöz, G., Varma, S., Sucu, S. (2016). Approximation by operators including generalized Appell polynomials. Filomat, 30(2), 429-440. DOI 10.2298/FIL1602429I.

15. Atakut, Ç., Büyükyazc, I. (2016). Approximation by Kantorovich-Szász type operators based on Brenke type polynomials. Numerical Functional Analysis and Optimization, 37(12), 1488-1502. DOI 10.1080/01630563.2016.1216447.

16. Tasdelen, F., Aktas, R., Altn, A. (2012). A Kantorovich type of Szász operators including Brenke-type polynomials. Abstract and Applied Analysis, 2012, 13. DOI 10.1155/2012/867203.

17. Varma, S., Sucu, S., Içöz, G. (2012). Generalization of Szász operators involving Brenke type polynomials. Computers \& Mathematics with Applications, 64(2), 121-127. DOI 10.1016/j.camwa.2012.01.025.

18. Çekim, B., Aktas, R., Içöz, G. (2019). Kantorovich-Stancu type operators including Boas-Buck type polynomials. Hacettepe Journal of Mathematics and Statistics, 48(2), 460-471. DOI 10.15672/HJMS.2017.528.

19. Usta, F. (2021). On approximation properties of a new construction of Baskakov operators. Advances in Difference Equations, 2021(269), 1-13. DOI 10.1186/s13662-021-03425-6.

20. Luo, Q. M. (2009). Q-extensions for the Apostol-Genocchi polynomials. General Mathematics, 17(2), $113-125$.

21. Araci, S., Khan, W. A., Acikgoz, M., Özel, C., Kumam, P. (2016). A new generalization of Apostol type Hermite-Genocchi polynomials and its applications. SpringerPlus, 5(860), 1. DOI 10.1186/s40064-016-2357-4.

22. Srivastava, M. H., Masjed-Jamei, M., Reza Beyki, M. (2018). A Parametric Type of the Apostol-Bernoulli, Apostol-Euler and Apostol-Genocchi Polynomials. Applied Mathematics \& Information Sciences, 12(5), 907-916. DOI 10.18576/amis/120502.

23. Prakash, C., Verma, D. K., Deo, N. (2021). Approximation by a new sequence of operators involving Apostol-Genocchi polynomials. Mathematica Slovaca, 71 (in Press).

24. Deo, N., Kumar, S. (2020). Durrmeyer variant of Apostol-Genocchi-Baskakov operators. Quaestiones Mathematicae, 44(4), 1-18. DOI 10.2989/16073606.2020.1834000.

25. Gadzhiev, A. D. (1976). Theorems of Korovkin type. Mathematical Notes of the Academy of Sciences of the USSR, 20, 995-998. DOI 10.1007/BF01146928.

26. Devore, R. A., Lorentz, G. G. (2005). Constructive approximation. Berlin: Springer-Verlag.

27. Johnen, H. (1972). Inequalities connected with the moduli of smootness. Matematicki Vesnik, 9(24), 289-305.

28. Davis, P. J. (1976). Interpolation and approximation. New York: Dover. 